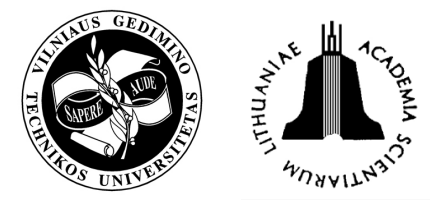

\title{
TECHNIQUE FOR DEFINITION OF BREAK SIZE OF ROAD COVERING ON THE BRIDGE
}

\author{
Jūratė Sužiedelytè-Visockienè \\ Dept of Geodesy and Cartography, Vilnius Gediminas Technical University, \\ Saulètekio al. 11, LT-10223 Vilnius, Lithuania \\ E-mail:j_visockiene@hotmail.com
}

Received 24 October 2006; accepted 1 February 2007

\begin{abstract}
The article deals with the submitted photogrammetric method for image rectification to the planar coordinate system. The images are taken by metric digital camera Canon EOS 350D. The camera is calibrated with Technet (Pictran $B, D / E$ ) software using the special test-field for calibration. Calibration parameters are determined to the images rectification process. The images in the planar coordinate system are transformed to 5 control points with the same software. The received results are visible in tables and figures. The transformed, planar images can be applied to research, designing and deformations estimate of engineering constructions.
\end{abstract}

Keywords: digital camera, camera calibration, planar model, image rectification, object deformation.

\section{Introduction}

The derivation of 2D (two-dimensional - planar) or 3D (three-dimensional) model from photographs or digital images is one of the main tasks of photogrammetry. The model consists of a cloud of space or planar points. 2D and 3D models are used for:

- topographic mapping;

- engineering and environmental applications;

- spatial data visualization;

- orthophotomap production;

- data integration into Geographic Information Systems (GIS) and combined analysis with other data.

The manual measurement of models with photogrammetric instruments is expensive and time consuming. Therefore the automation of $2 \mathrm{D}$ or $3 \mathrm{D}$ reconstruction from aerial photographs, satellite images and digital images is urgently needed.

In this article 2D model reconstruction technology using digital images is explained.

Basic processes of creation 2D model from images are:

- project creation - creating the project definition, the project directory and establishing the coordinate system, datum, ground units;

- imagery - digital images taken;
- camera calibration - computing of digital camera parameters;

- ground control point (GCP) measurement, i. e. geodetic measurement;

- image rectification to the planar coordinate system;

- data importation to Cad software;

- digitalisation of object in the planar image;

- data analysis.

2D model is often used for construction engineering projection, object deformation observation, accident reconstruction in the traffic. These approaches represent the experiment of digital images rectification on purpose to follow the deformation of road covering on the bridge. It is necessary to find the break area of the road.

\section{Process of camera calibration and image rectification}

Camera calibration. The camera lens system (model) consists of 6 parameters: $A_{1}, A_{2}, A_{3}$ - for radial symmetrical and $B_{1}, B_{2}, B_{3}$ - for radial asymmetrical distortions. The radial symmetric model requires the definition (or estimation) of a center point $\left(x_{0}, y_{0}\right)$. We have fixed this point in the center of images. The polynomials for radial symmetric distortion are $[1,2]$ : 


$$
\left.\begin{array}{l}
d_{x}=A_{1} x_{0}\left(r^{2}-r_{0}^{2}\right)+A_{2} x_{0}\left(r^{4}-r_{0}^{4}\right)+A_{3} x_{0}\left(r^{6}-r_{0}^{6}\right) ; \\
d_{y}=A_{1} y_{0}\left(r^{2}-r_{0}^{2}\right)+A_{2} y_{0}\left(r^{4}-r_{0}^{4}\right)+A_{3} y_{0}\left(r^{6}-r_{0}^{6}\right) ; \\
r^{2}=\left(\frac{d_{x}}{s_{x y}}\right)^{2}+d_{y}^{2},
\end{array}\right\}
$$

where $x_{0}, y_{0}$ - coordinate of image center point (principal point); $A_{1}, A_{2}, A_{3}$ - radial symmetric distortion; $S_{x y}$ - image scale constant; $r_{0}$ - constant introduced to reduce the correlation between the distortion coefficients and principal distance $c$ (focal length of camera objective). Generally this constant is $1 / 3$ in length of the diagonal size of the image. $[1,2]$ :

The radial asymmetric distortions are described by

$$
\begin{aligned}
& d_{x}=B_{1}\left(r^{2}+2 x_{0}^{2}\right)+B_{2} 2 x_{0} y_{0} \\
& d_{y}=B_{2}\left(r^{2}+2 y_{0}^{2}\right)+B_{3} 2 x_{0} y_{0},
\end{aligned}
$$

where $B_{1}, B_{2}, B_{3}$ - parameters of radial asymmetric distortions.

For the camera calibration the user has to provide images of the test-field, taken by the camera-system from different positions and with different orientations (Fig 1). The test-fields have the retro reflective points with known coordinates $(x, y, z)$. The test-fields points are detected by means of manual, automatic or semiautomatic measurement methods with software.

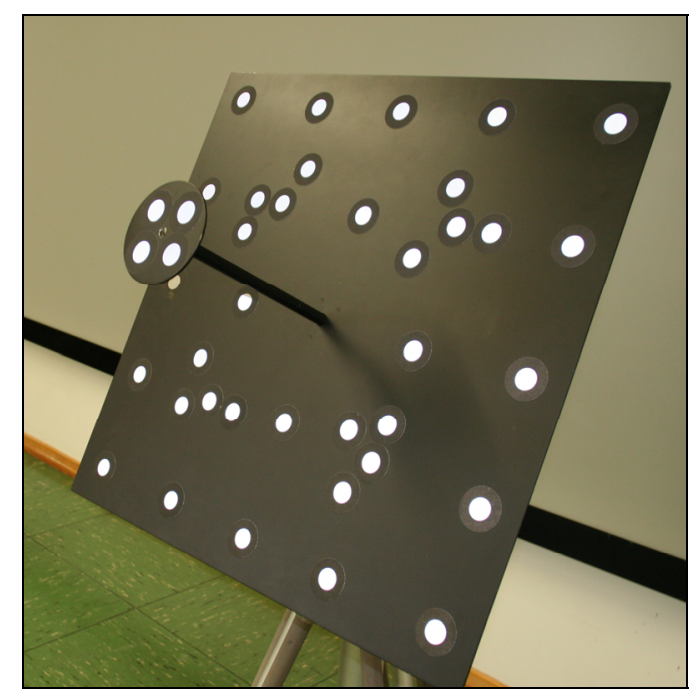

Fig 1. The test-field of camera calibration

Parameters of camera calibration are obtained after calculation of polynomials $(1,2)$.

The accuracy of camera calibration results depends on several factors:

- accuracy of image point measurement: - camera capability (stability, calibration);
- measurement accuracy of evaluation system (measurement algorithm);

- point adjustability (recognizability, marking);

- mapping scale;

- photograph configuration;

- control point accuracy.

Image rectification. Prerequisite for rectification is the planarity of object (facades, paintings, under floor heating, object deformations, etc.). There are at least 4 control points within this planar necessary to determine the 8 parameters of rectification; the image can then be reprojected. Rectification result could be a drawing or an image map. The destination image can be processed by one or more initial images.

For every control point two equations are calculated $[3,4]$ :

$$
\left.\begin{array}{l}
X=\frac{A_{1} x+A_{2} y+A_{3} z}{C_{1} x+C_{2} y+1} \\
Y=\frac{B_{1} x+B_{2} y+B_{3} z}{C_{1} x+C_{2} y+1}
\end{array}\right\}
$$

where $X, Y$ - control points coordinate transformed to the plane.

$$
\left.\begin{array}{l}
A_{1}=Z \frac{a_{1}}{c_{3} z} ; A_{2}=Z \frac{a_{2}}{c_{3} z} ; A_{3}=Z \frac{a_{3}}{c_{3}} ; \\
B_{1}=Z \frac{b_{1}}{c_{3} z} ; B_{2}=Z \frac{b_{2}}{c_{3} z} ; B_{3}=Z \frac{a_{3}}{c_{3}} ; \\
C_{1}=\frac{c_{1}}{c_{3} z} ; C_{2}=\frac{c_{2}}{c_{3} z} .
\end{array}\right\}
$$

In the equations (3), (4) $x, y, z$ - known coordinate of control points, in object coordinate system; $A_{1}$, $A_{2}, A_{3}, B_{1}, B_{2}, B_{3}, C_{1}, C_{2}-8$ unknown parameter transformation; $a_{i}, b_{i}, c_{i}$ - rotation of images.

If you know parameters transformation, you get calculated coordinates of every point.

Rectification can still be performed without loss of accuracy, if the coordinates of the principal point $\left(x_{0}, y_{0}\right)$ and the principal distance $c$ are unknown. Influences of lens distortion are not covered by the unknowns and should be determined in advance by a camera calibration in order to consider them while making calculations.

Digital rectification has advantages over the analogue reprojection:

- even image with extreme viewing angle can be rectified without significant losses in details;

- camera lens distortions can be easily corrected;

- by using image filtering techniques the image can be enhanced and details can be outlined. 


\section{Estimate of the road covering deformation on the bridge}

Research was carried out in Vilnius Gediminas Technical University (VGTU), Photogrammetry Laboratory of Geodesy Institute. Test object was the road covering on the bridge in the city of Vilnius. In the middle of the bridge the break of road was observed. We needed to estimate the size of break.

The two images were taken in June 2006 with metric digital photo camera Canon EOS 350D (Fig 2).

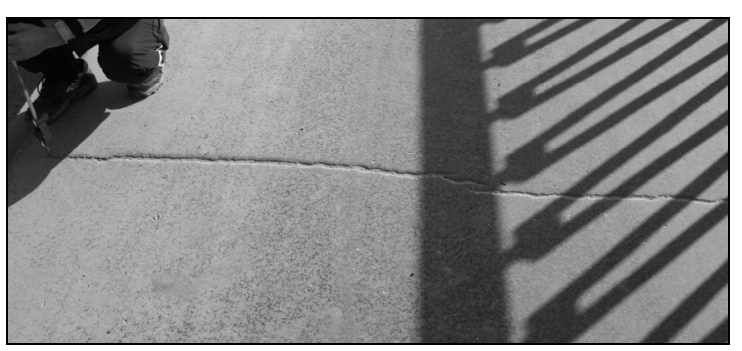

Fig 2. One image of break on the bridge

The camera was calibrated in Photogrammetry laboratory of Geodesy Institute in VGTU, with software Pictran (Technet, Germany). The camera's parameters and precision of calibration are presented in Table 1.

Table 1. Results of camera Canon EOS 350D calibration

\begin{tabular}{|c|c|}
\hline Parameters & $\begin{array}{c}\text { Result of camera calibration, } \\
\mathrm{mm}\end{array}$ \\
\hline \multicolumn{2}{|c|}{ Focal length of camera objective } \\
\hline$c$ & 21,116637 \\
\hline$S_{x y}$ & 1,65785 \\
\hline \multicolumn{2}{|c|}{ Coordinate of image center point } \\
\hline$x_{0}$ & $-0,586128$ \\
\hline$y_{0}$ & 0,821965 \\
\hline \multicolumn{2}{|c|}{ Symmetric distortion } \\
\hline$A_{1}$ & $-0,00021$ \\
\hline$A_{2}$ & 0,00000047 \\
\hline$B_{1}$ & $0,8690 \times 10^{-4}$ \\
\hline$B_{2}$ & $-0,3684 \times 10^{-3}$ \\
\hline \multicolumn{2}{|c|}{ Precision rate of camera calibration } \\
\hline$\sigma, \mu \mathrm{m}$ & 0,5455 \\
\hline
\end{tabular}

Value $\sigma$ is standard deviation of weight unit after the calculation. With this value you can check, if the standard deviation of the observation is chosen correctly. If $0,5<\sigma<1,3$ is guaranteed, that model is correct, that means you have applied correct standard deviations for the observations $[4,5]$. In the research the value for $\sigma$ is $0,54 \mu \mathrm{m}$, it is within acceptance range.

Around the object there are 5 control points. Coordinates of these points are measured by geodetic method in the local coordinate system (Table 2).

These control points were measured manually in the two images with software Pictran. After image rectification the control point coordinate in the image coordinate system was obtained and a model of the planar coordinate system was created (Fig 3).

In Table 3 images rectification protocol is presented.

Table 2. Coordinates of control points in the object

\begin{tabular}{|c|c|c|c|}
\hline \multirow{2}{*}{$\begin{array}{c}\text { Number of } \\
\text { control point }\end{array}$} & \multicolumn{3}{|c|}{ Coordinate, $\mathrm{m}$} \\
\cline { 2 - 4 } & $x$ & $y$ & $z$ \\
\hline 1 & 520,718 & 515,987 & 100,248 \\
\hline 2 & 520,767 & 517,113 & 100,255 \\
\hline 3 & 520,639 & 517,379 & 100,257 \\
\hline 4 & 520,593 & 518,198 & 100,262 \\
\hline 5 & 520,537 & 518,900 & 100,264 \\
\hline
\end{tabular}

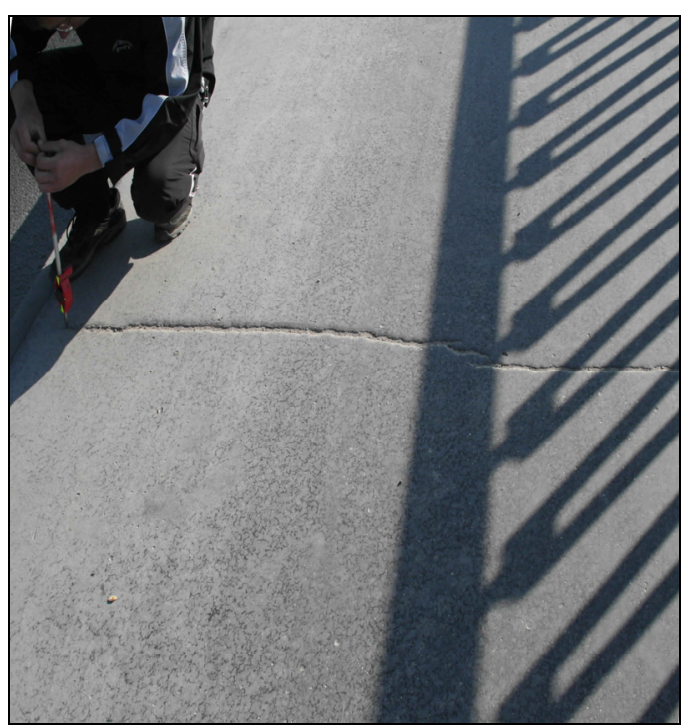

Fig 3. Rectified image

Table 3. Images rectification protocol

\begin{tabular}{|c|c|c|}
\hline \multirow{2}{*}{$\begin{array}{c}\text { Number of GCP } \\
\text { point }\end{array}$} & $\begin{array}{c}\text { Control point coordinate in the image } \\
\text { coordinate system, mm }\end{array}$ \\
\cline { 2 - 3 } & $x$ & $y$ \\
\hline 1 & $-8,3130$ & 1,1217 \\
\hline 2 & 1,9761 & 1,2422 \\
\hline 3 & 4,5712 & 0,3830 \\
\hline 4 & 2,9960 & 0,3045 \\
\hline 5 & 9,5057 & 0,3777 \\
\hline \multicolumn{3}{|c|}{$\delta_{0}=0,0010$} \\
\hline
\end{tabular}

Accuracy of point measurement in the digital images is $\delta_{0}=10 \mu \mathrm{m}$. It is high precision.

2D model evaluation. Planar object model can be imported to the next Cad software (ACad, MicroStation, ArcGis, etc), digitising and measuring the size of break. The test model was imported to the ACad program. The length and width of object were digitised. Length is $60,6 \mathrm{~cm}$, width $-0,08 \mathrm{~cm}$. Area of break is about $4,848 \mathrm{~cm}^{2}$ (Fig 4). 


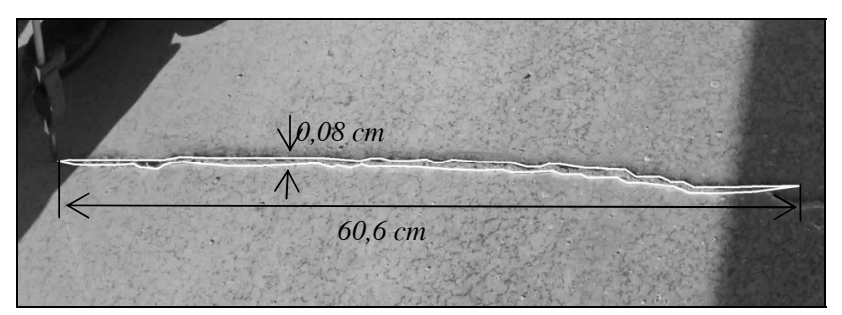

Fig 4. Measurement of the break of road covering

In practice it is interesting to observe deformation of an object. It takes a short time to take images, to measure and document the results of deformation. Also, man can evaluate a risk and how to deal with this situation.

\section{Advantages of this method}

Photogrammetry is an efficient procedure which distinguishes itself by several advantages:

1. High accuracy: even with little effort the high point of accuracy can be achieved.

2. Short object coverage time: it is not necessary to stay longer at the object which is to be covered.

3. Huge amount of object information: with images it is possible to obtain a huge amount of object information in a short time. The image contains object geometry and is reconstructionable.

4. Objects and details are permanently stored on images. The evaluation of images and the analysis of results can be performed years after the actual picture taking.

\section{References}

1. ABRAHAM, S., HAU, T. Towards autonomous highprecision calibration of digital cameras. In Proceedings of SPIE Annual Meeting, 1997, San Diego, SPIE, Vol 3174, p. 82-93.

2. MARZAN, G. T.; KARARA, H. M. A computer program for direct linear transformation solution of the colinearity condition, and some applications of it. In ASP Symposium on Close-range Photogrammetric Systems. Falls Church, VA: American Society of Photogrammetry, 2003, p. 420-476.

3. Pictran-B. Program description and user manual. $121 \mathrm{p}$.

4. Pictran-D/E. Program description and user manual. $105 \mathrm{p}$.

5. LÄBE, TH.; FÖRSTNER, W. Geometry stability of lowcost digital consumer cameras. Institute für Photogrammetrie, Universität Bonn, Commission I/2, 2004. $7 \mathrm{p}$. 